MODELING, IDENTIFICATION AND CONTROL, 1991, VOL. 12, NO. 1, 27-46

doi:10.4173/mic.1991.1.3

\title{
Transient simulations of gas-oil-water separation plants
}

\author{
TOR STEINAR SCHEI†, PETER SINGSTAD \\ and AAGE JOSTEIN THUNEM
}

Keywords: Dynamic simulation, oil processing, oil separation.

\begin{abstract}
A set of mathematical models for the dynamic simulation of offshore processing plants is developed. Each process unit is modeled separately, and the various models are integrated into a system for the simulation of an entire plant. The purpose of the simulation system is to study the effects of various disturbances and investigate appropriate control strategies. Important variables subject to control are pressure, flow rate, temperature, vessel liquid level and compressor speed. In separators the rate of interfacial mass transfer between the liquid and vapour phases at nonequilibrium is modeled as a first order time lag. The vapour-liquid equilibrium ratio is linearized with respect to variations in pressure and temperature for each separator stage. A realistic scenario is chosen in order to demonstrate the capabilities of the simulation system.
\end{abstract}

\section{Introduction}

Process simulation systems are important tools in process design, control system design and plant operation analysis. The following application areas are typical:

Static and dynamic analysis of various plant configurations.

Design of process components and unit operations. Each unit operation can be optimized with respect to overall plant performance.

Testing of control system designs as a tool for structure and parameter selection. Factory test of turnkey control systems.

Operational simulators used by the system operator in order to test planned operations or as an exploratory aid in testing different operational strategies.

Study the consequences of component failures.

Study start-up and shut-down procedures.

Training simulators for the training of plant operators.

An offshore processing plant for crude oil is subject to various disturbances in crude flow rate, vapour-liquid ratio and crude temperature. These disturbances are the consequences of planned actions, component failures or variations in flow regime. Slug flow is probably the most serious disturbance. The start-up and shut-down of the entire plant or parts of it also represent severe process disturbances.

During the start-up of subsea wells, crude temperatures can vary from about $0^{\circ} \mathrm{C}$ to $100^{\circ} \mathrm{C}$ in a few minutes. These temperature variations disturb the vapour-liquid equilibrium ratios and influence the performance of the heat-exchangers.

During various process disturbances it is important to optimize the control of pressure, flow rate, temperature and vessel liquid level.

Received 20 December 1990.

† SINTEF Automatic Control, N-7034 Trondheim, Norway. 
SINTEF Automatic Control has developed dynamic models for separators, valves, heat exchangers, scrubbers, compressors and turbines. The various models are integrated into a system for the simulation of an entire plant.

The purpose of the simulation system is to study the dynamic behaviour of the plant under different operational conditions, and furthermore investigate the influence of various process disturbances and control strategies on plant performance. This system is also an efficient tool to evaluate plant performance for design purposes. The effect of the size and characteristics of equipment on plant performance can be studied. The simulation model is also used to consider the sensitivity of various process parameters on plant performance.

Computer simulations show that the dynamic interactions between the separators and the compressor modules are significant. Hence, it is also necessary to include a model of the compressor system in order to obtain realistic simulation conditions for the separator train.

Some general references on gas-oil-water separation are Arnold and Stewart (1987a and b) and Callaghan et al. (1985). The dynamic models for the various process units are based on Bird et al. (1960), Campbell (1984), Lydersen (1985) and Wu (1987).

\section{Liquid-vapour mass transfer}

The interfacial mass transfer rate between the liquid and vapour phase has been assumed to be proportional to the 'distance from equilibrium' for the amount of gas in liquid solution.

$$
R_{s f}=c\left(\gamma_{s}-\gamma_{s, \mathrm{eq}}\right)
$$

$\boldsymbol{R}_{s f}$ is the mass transfer rate per unit volume $\left(\mathrm{kg} / \mathrm{m}^{3} \mathrm{~s}\right), \gamma_{s}$ is the mass of gas in solution per unit liquid volume $\left(\mathrm{kg} / \mathrm{m}^{3}\right)$ and $\gamma_{s, \text { eq }}$ is the same quantity at equilibrium.

The equation has been chosen because of its simplicity and from its analogy with the common practice of modeling component flux across an interfacial film (Lydersen, 1985).

The constant, $c$, in Eqn. (1) is dependent on fluid flow conditions, fluid composition, free interfacial area and the geometry of the vessel. In order to calculate the equilibrium $\gamma_{s, \text { eq }}$ in Eqn. (1) as a function of time-varying pressure and temperature, the following ratio is defined.

$$
X=\left.\frac{\gamma_{s}}{\rho_{l}}\right|_{\text {equilibrium }}=\frac{\gamma_{s, \text { eq }}}{\rho_{l}}
$$

where $\rho_{l}$ is the liquid density $\left(\mathrm{kg} / \mathrm{m}^{3}\right)$.

The ratio $X$ is a function of pressure, temperature and composition of the fluid in the current control volume. In the dynamic simulations, a linearized equation for $X$ is used:

$$
X=X_{\mathrm{q}}+X_{\mathrm{p}} \cdot\left(P-P_{\mathrm{q}}\right)+X_{\mathrm{T}} \cdot\left(T-T_{\mathrm{q}}\right)
$$

Here $X_{\mathrm{q}}, X_{\mathrm{p}}, X_{\mathrm{T}}, P_{\mathrm{q}}$ and $T_{\mathrm{q}}$ are constants. To account for varying fluid composition, one set of these constants must be calculated for each separator stage.

The constants in Eqn. (3) are calculated in conjunction with a standard flash calculation procedure. The following equations are derived by partial derivation of $X$ with respect to pressure and temperature.

$$
X_{\mathbf{q}}=1-\frac{L^{m} M_{l}^{m}}{L M_{l}}
$$




$$
\begin{gathered}
X_{\mathrm{p}}=\frac{F}{(L)^{2} M_{l}} L^{m} M_{l}^{m}(-1) \frac{\sum_{i=1}^{n} \frac{z_{i}}{\left(\xi k_{i}+1-\xi\right)^{2}} \frac{\partial k_{i}}{\partial p}}{\sum_{i=1}^{n} \frac{-z_{i}\left(k_{i}-1\right)^{2}}{\left(\xi k_{i}+1-\xi\right)^{2}}} \\
X_{\mathrm{T}}=\frac{F}{(L)^{2} M_{l}} L^{m} M_{l}^{m}(-1) \frac{\sum_{i=1}^{n} \frac{z_{i}}{\left(\xi k_{i}+1-\xi\right)^{2}} \frac{\partial k_{i}}{\partial T}}{\sum_{i=1}^{n} \frac{-z_{i}\left(k_{i}-1\right)^{2}}{\left(\xi k_{i}+1-\xi\right)^{2}}}
\end{gathered}
$$

Superscript $m$ is used for variables referring to the last separator stage. All other variables refer to the current stage.

$$
\begin{aligned}
\xi & =\frac{V}{F} \\
k_{i} & =\frac{y_{i}}{x_{i}}
\end{aligned}
$$

In this study, the equilibrium constants $k_{i}$ reported by Standing (1979) are used. The rate of condensation inside heat exchangers is modeled in a slightly different way. Outlet liquid and vapour are considered to be in equilibrium at a specific pressure and temperature.

$$
w_{l, o}=w L\left(P, T_{o}\right)
$$

$L$ is the equilibrium mass fraction of liquid in the outlet flow, this is a function of pressure and temperature. Variations in pressure and temperature are accounted for by linearizing around the steady state.

\section{Dynamic models}

\subsection{Two-phase vapour-liquid separator}

The mass and energy balance for a separator is given by a dynamic model with lumped parameters. The inlet crude is described as a two-phase fluid with a variable vapour-liquid ratio and a variable concentration of dissolved gas in oil. At the outlet the gas-oil ratio will vary with the vessel condition, geometry and internals used.

The rate of mass transfer between the liquid and vapour phase is modeled as a first order process. That is; the rate of mass transfer is proportional to the 'distance from equilibrium' at the specific pressure and temperature.

The equations below describe the mass and energy balance for a vapour-liquid separation vessel.

Mass transfer

$$
R_{s, f}=c\left(m_{s}-X(P, T) m_{l}\right)
$$

Mass balance

$$
\begin{gathered}
\dot{m}_{l}=w_{l, i}-w_{l, o}-R_{s, f} \\
\dot{m}=w_{s, i}-w_{s, o}-R_{s, f} \\
\dot{m}=w_{v, i}-w_{v, o}+R_{s, f}
\end{gathered}
$$


At the separator outlet, the liquid and vapour are considered to be in equilibrium at the particular pressure and temperature. That is,

$$
w_{s, o}=X(P, T) \cdot w_{l, o}
$$

Energy balance

$$
\dot{U}=w_{v, i} h_{v, i}+w_{l, i} h_{l, i}-w_{v, o} h_{v}-w_{l, o} h_{l}
$$

The vessel is considered to be thermally insulated.

Equations of state

$$
\begin{aligned}
U= & H-P V \\
h_{l}= & h_{q l}\left(T_{q} ; M_{l}\right)+c_{p l}\left(M_{l}\right) \cdot\left(T-T_{q}\right) \\
h_{v}= & h_{q l}\left(T_{q} ; M_{l}\right)+h_{e v}\left(T_{q}, P_{q}\right) \\
& +c_{p v}\left(M_{v}\right) \cdot\left(T-T_{q}\right)+C_{t}\left(M_{v}\right) \cdot\left(P-P_{q}\right) \\
V_{L}= & \frac{m_{l}}{\rho_{l}} \\
P= & \frac{m_{v}}{M_{v} \cdot\left(V-V_{L}\right)} z R T \\
H= & m_{l} \cdot h_{l}+m_{v} \cdot h_{v}
\end{aligned}
$$

Specific enthalpy (Eqns. 17 and 18) is approximated as a linear function of pressure and temperature around the steady state. This approximation is very close to the real values for realistic operating conditions.

The liquid is assumed to be incompressible. From an energy point of view this assumption should be satisfactory. The $z$-factor in Eqn. 20 is approximated as a constant.

Scrubbers are modeled in essentially the same way as the two-phase separators.

\subsection{Gas-oil-water separator}

Liquid inside the separator is considered to be divided into two separate layers. Inlet crude enters the upper layer of oil and water drops. Some of the water drops separate from the oil phase and enter the lower layer of free water. Gas-oil and oilwater interface levels are controlled with separate liquid level controllers.

The mass and energy balance for the gas and oil phase is described in the previous section. Gas-oil equilibrium conditions and the interfacial mass transfer are considered to be independent of the water content in crude. That is a common assumption when calculating the vapour-liquid equilibrium for hydrocarbons.

The equations below describe the mass balance for water drops in oil and free water in the separator vessel. The quantity of separated water per time unit is assumed to be proportional to the quantity of water drops in the oil phase and inversely proportional to the oil phase thickness.

Water separation

$$
R_{d, w}=C_{w} m_{d} /\left(l_{o}-l_{w}\right)
$$


Mass balance

$$
\begin{aligned}
\dot{m}_{d} & =W_{w, i}-W_{w, l o}-R_{d, w} \\
\dot{m}_{w} & =R_{d, w}-W_{w, o} \\
x_{w} & =m_{d} /\left(m_{d}+m_{o}\right) \\
W_{w, l o} & =x_{w} W_{l, l o}
\end{aligned}
$$

\subsection{Heat exchanger}

Heat exchangers are typical examples of distributed parameter systems. For a detailed study of heat exchanger dynamics it is necessary to use a model that is at least distributed in the main flow direction. Since the objective was the study of the overall system dynamics and not heat exchanger performance in particular, we used a lumped parameter model of heat exchangers to save computational time.

A contracurrent tube-and-shell heat exchanger was chosen. As the main disturbance is variation in the primary inlet flow and to some extent the inlet temperature, the heat exchanger dynamic model was developed accordingly.

The condensation rate is given by Eqn. (9).

$$
\begin{aligned}
\dot{T}_{o} & =\frac{w}{m}\left(T_{i}-T_{o}\right)-\frac{Q}{m C_{p}}+\frac{h_{e v}}{m C_{p}}\left(w_{l, o}-w_{l, i}\right) \\
\dot{T}_{c, o} & =\frac{w_{c}}{m_{c}}\left(T_{c, i}-T_{c, o}\right)+\frac{Q}{m_{c} C_{p c}} \\
Q & =U \cdot A \cdot \Delta T_{m} \\
\Delta T_{m} & =\frac{1}{2}\left(T_{i}+T_{o}\right)-\frac{1}{2}\left(T_{c, i}+T_{c, o}\right)
\end{aligned}
$$

\subsection{Compressor}

The compressor model was developed assuming an adiabatic polytropic compression process. This model uses a performance diagram where normalized volume flow is expressed as a function of the normalized pressure ratio where the normalized compressor speed is a parameter. The performance diagram is specific for each compressor. That is, the pressure-flow relation has to be measured from the actual compressor. Even if compressors are produced after the same specifications, their performance diagrams will not be identical. This diagram is also dependent on the compressor's guide vane position and the gas density. Figure 1 shows a typical pressure-flow performance diagram for a compressor. The compressor model is based on Lapina (1982) and Straroselsky (1979).

Compressor efficiency can be expressed as

$$
\eta=\frac{n}{n-1} / \frac{k}{k-1}
$$

where $k$ is the isentropic and $n$ the polytropic exponent. $\eta$ can be correlated against compressor speed, and volume flow. Given $\eta$ and $k, n$ can be computed. An equation for the change in enthalpy through the compressor (also called polytropic head) can be deduced from elementary thermodynamic equations, and by applying Eqn. 31 we get

$$
h=\frac{z R T_{1}}{M} \frac{k \eta}{k-1}\left[f^{k-1 / \eta \cdot k}-1\right]
$$




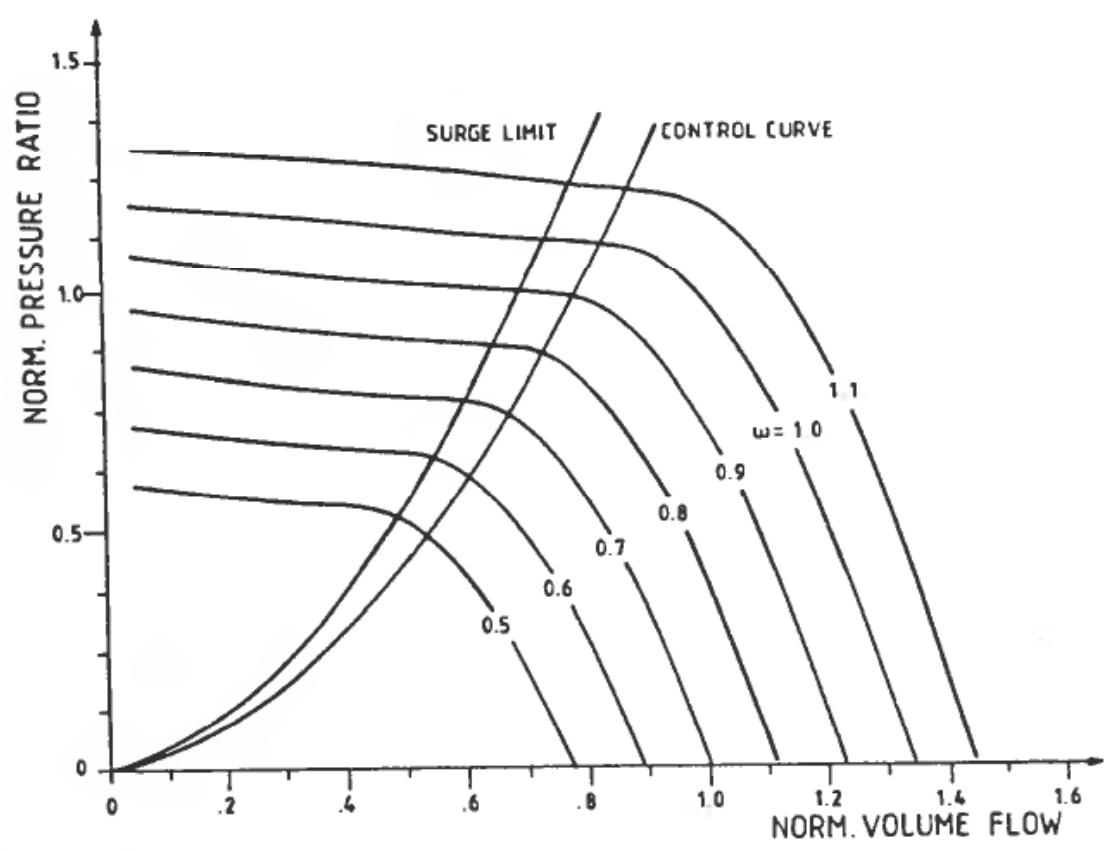

Figure 1. Pressure-flow performance diagram for a compressor, $\omega$ is normalized compressor speed.

The outlet gas temperature is

$$
T_{2}=T_{1} \cdot f^{k-1 / \eta k}
$$

The compressor power consumption is

$$
B_{c}=\frac{h w}{\eta}
$$

The compressor surge limit curve is assumed to be parabolic in the compressor performance diagram.

\subsection{Gas turbine}

A gas turbine is used to drive one or more compressors working on the same shaft. The shaft power balance:

$$
B_{T}-\sum_{i} B_{c i}-B_{F}=I \Omega \frac{d \Omega}{d t}
$$

There is a time lag between the position of the valve controlling the fuel supply and the turbine power generation. Turbine efficiency is also speed dependent.

$$
\begin{gathered}
B_{T}=\frac{2 \pi}{60} N C_{T}\left(2-\frac{N}{N_{0}}\right) T_{0} \\
\dot{C}_{T}=\frac{1}{T}\left(u-C_{T}\right), C_{T} \in[0,1]
\end{gathered}
$$

Friction loss is given by

$$
B_{F}=C_{F} N^{2}
$$




\section{Controller tuning}

The tuning of ordinary PID-controllers is theoretically a simple task providing a relevant dynamic model of the process to be controlled is available. However, in practice this is not a trivial matter at all. Controllers are often tuned by experimenting with the process and without using analytical methods. Even if only proportional and integral controller action is utilized it can be extremely difficult to obtain good controller tuning this way. The result is often poor controller performance and much lower bandwidth than should be necessary.

This section consequently includes some rules of thumb for PID-controller tuning. The controller parameters have been calculated from the dynamic models in the previous section by using ordinary frequency analytical techniques for single loops. The nonlinear models are linearized around stationary operating conditions so that linear theory can be applied.

\subsection{Level controller}

The loop transfer function for the separator level control with proportional and integral action is

$$
\begin{gathered}
h_{l}(s)=\frac{k_{p} k\left(1+T_{i} s\right)}{T_{i} s^{2}\left(1+T_{v} s\right)} \\
k=\frac{k_{v}}{A}\left(\frac{\Delta p}{\rho_{1}}\right)^{1 / 2}
\end{gathered}
$$

It is assumed that the valve characteristic is linear and that the pressure difference across the valve is independent of the volume flow.

To get good stability, the integral time $\left(T_{i}\right)$ should be set to at least ten times the valve operation time $\operatorname{lag}\left(T_{v}\right)$. The system bandwidth should be in the interval from $1 / T_{i}$ to $1 / T_{v}$; and the maximum stability margin is obtained for

$$
\omega_{c}=\left(T_{i} T_{v}\right)^{-1 / 2}
$$

To get the right bandwidth the controller proportional constant has to be set to

$$
k_{p}=\omega_{c} / k
$$

If it is desirable to reduce controller bandwidth in order to reduce downstream disturbances, integral time should be set to a higher value and $k_{p}$ adjusted in accordance with Equations 41 and 42. It is worth noting that a too low value of $k_{p}$ like a too high value will reduce the stability margin.

\subsection{Pressure controller}

The loop transfer function for separator pressure control with proportional and integral action is

$$
\begin{aligned}
h_{p}(s) & =\frac{k_{p} k\left(1+T_{i} s\right)}{T_{i} s\left(1+T_{v} s\right) k_{f}\left(1+\frac{1}{k_{f}} s\right)} \\
k & =\frac{z R T}{M_{v} V_{v}} k_{v}\left(\Delta_{p} \rho_{v}\right)^{1 / 2} \\
k_{f} & =\frac{z R T}{M_{v} V_{v}} c m_{l} X_{p}
\end{aligned}
$$


It is assumed that the valve characteristic is linear and that the pressure difference across the valve is independent of the volume flow. $X_{p}$ is the equilibrium pressure sensitivity. To get a good stability margin, the integral time and proportional constant can be set to

$$
\begin{aligned}
& T_{i} \approx 10 T_{v} \\
& k_{p}=\frac{M_{v} V_{v}}{z R T T_{v} k_{v}\left(\Delta_{p} \rho_{v}\right)^{1 / 2}}
\end{aligned}
$$

\section{A simulation scenario}

A part of a processing plant for crude oil is modeled and simulated. A simplified process diagram is shown in Fig. 2. The plant is designed with two separator trains in parallel. Each train processes fifty per cent of the total production. There are also two compressor trains, leaving one as a stand-by.

One separator train is simulated dynamically. The other is assumed to be in a stationary state. The reason for this is that the two separator trains are connected to different wells and it is assumed that they were not upset at the same time. The dynamics of the first and second stage compressor systems are also neglected in the simulation model. Output pressure from the fifth stage compressor is constant.

Conventional control structures and algorithms are applied.

Initially, the plant operates in a stationary state. After 30 seconds of simulation it is disturbed by a $50 \%$ increase in inlet flow (Fig. 3). After another 90 seconds the inlet flow decreases to the normal rate.

Figures 3 to 6 show the liquid inlet and outlet flow rate for all four separator stages. The disturbance generated in the first stage is transferred to the other stages and is smoothed out somewhat. It is difficult to tune the controllers in a way that will reduce

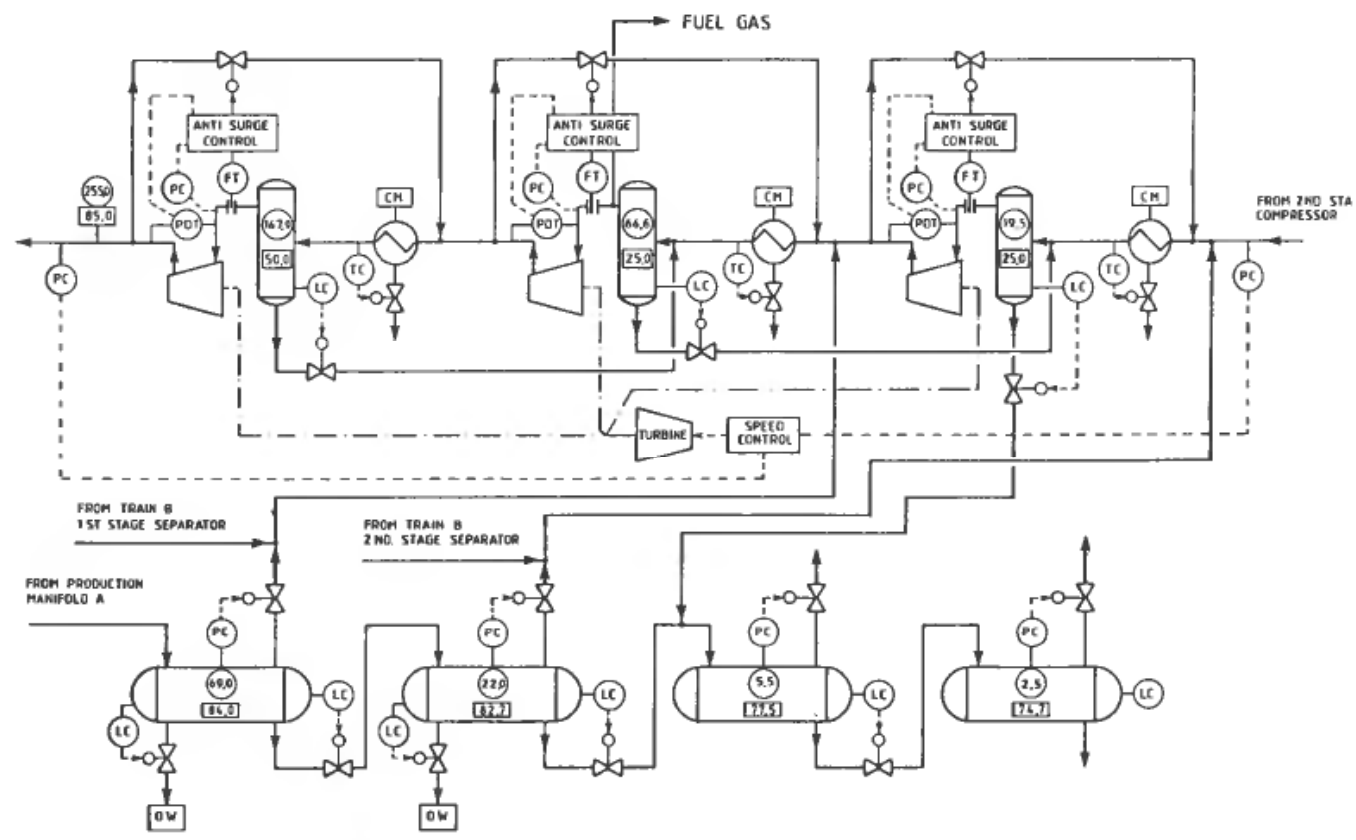

Figure 2. Part of a processing plant for crude oil. 


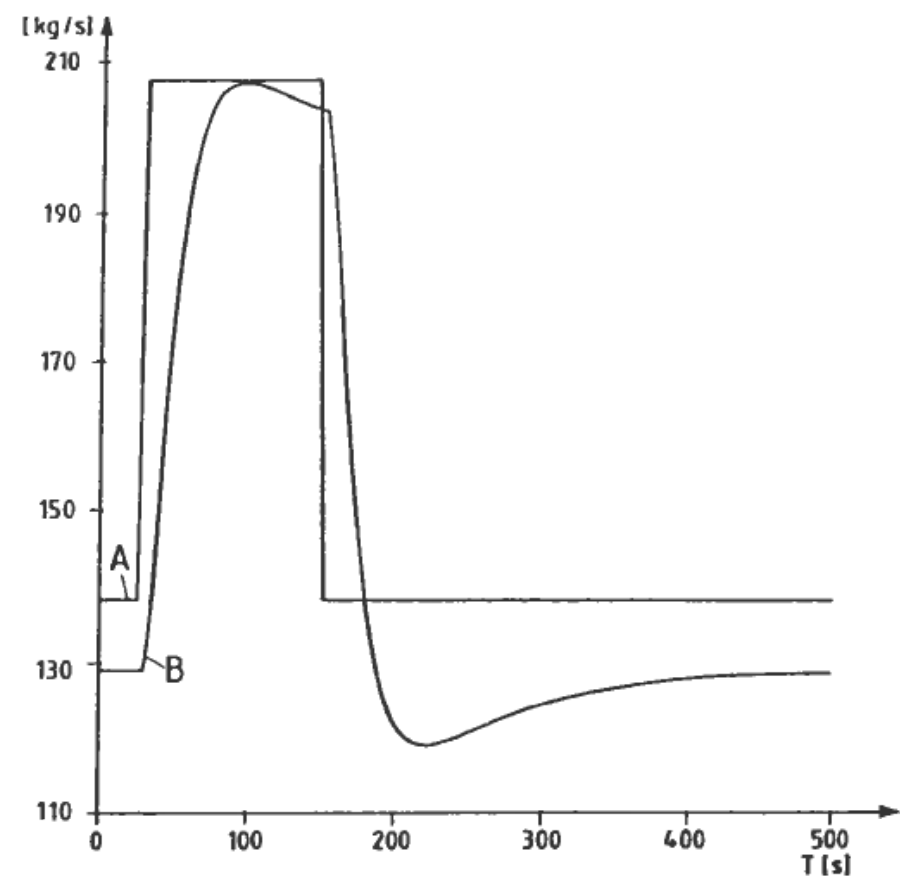

Figure 3. First separator stage inlet (A) and oil outlet (B) liquid flow rate.

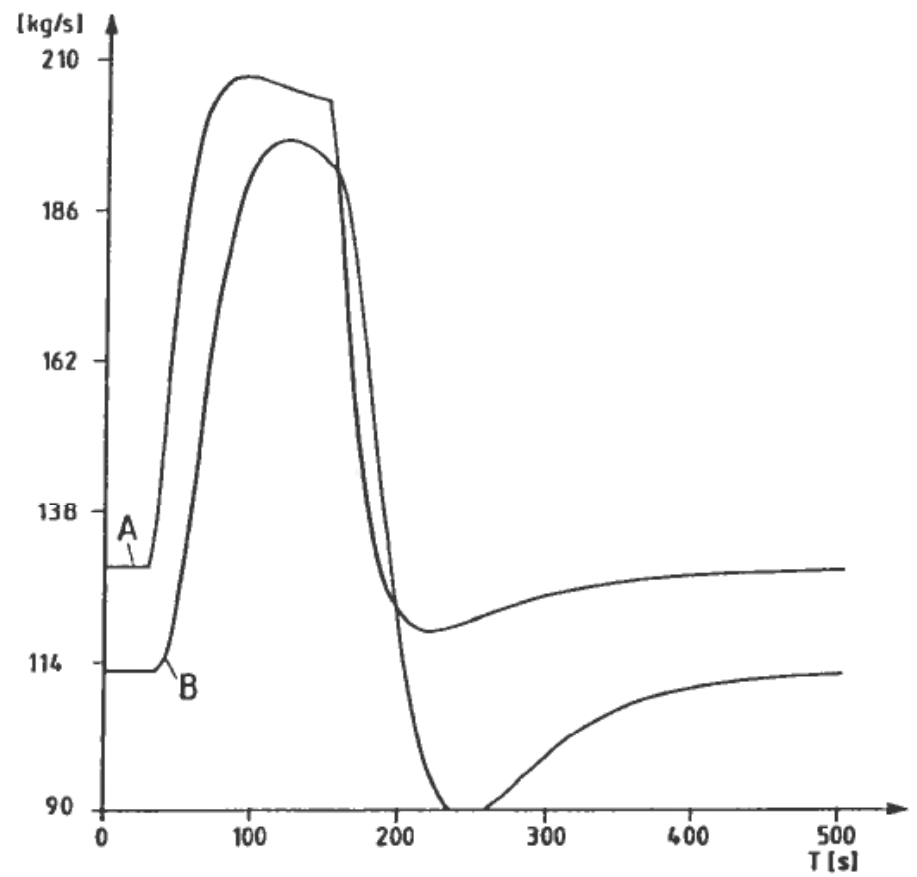

Figure 4. Second separator stage inlet (A) and oil outlet (B) liquid flow rate. 
T. S. Schei et al.

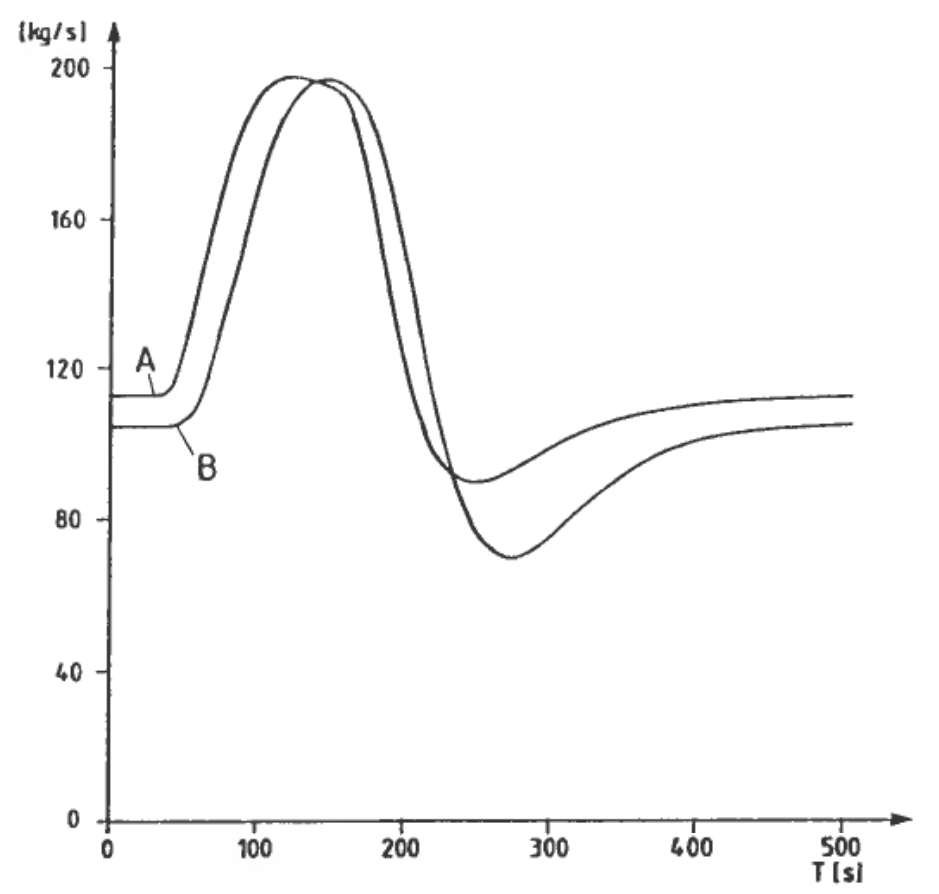

Figure 5. Third separator stage inlet (A) and outlet (B) liquid flow rate.

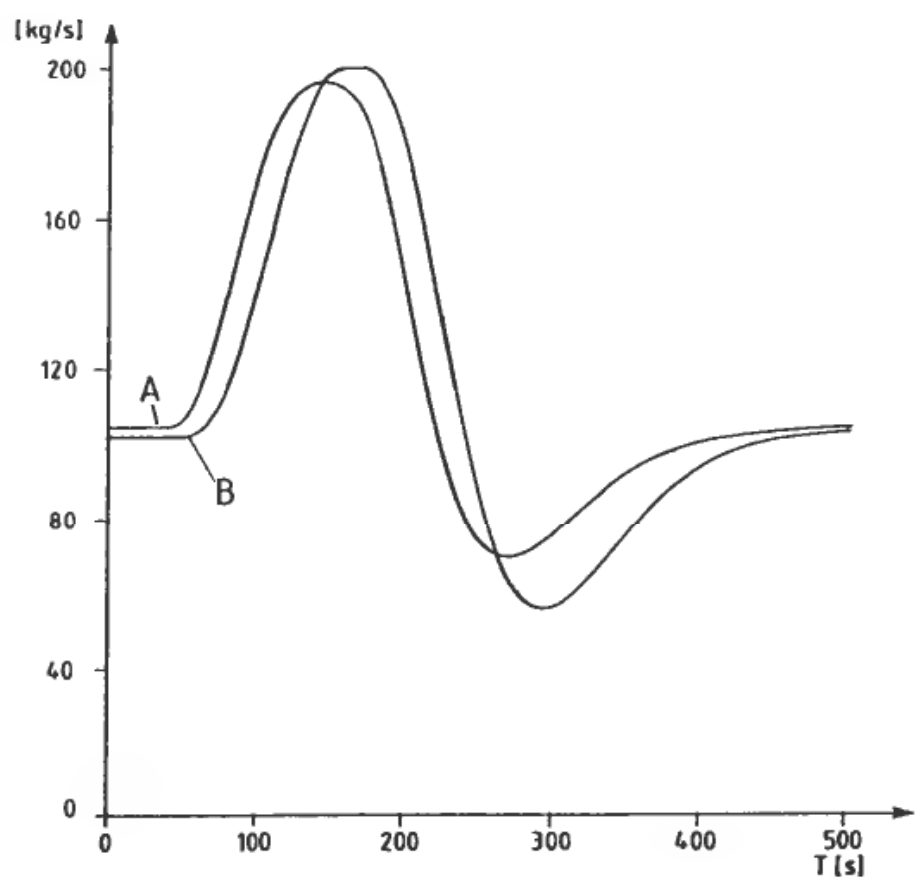

Figure 6. Fourth separator stage inlet (A) and outlet (B) liquid flow rate. 


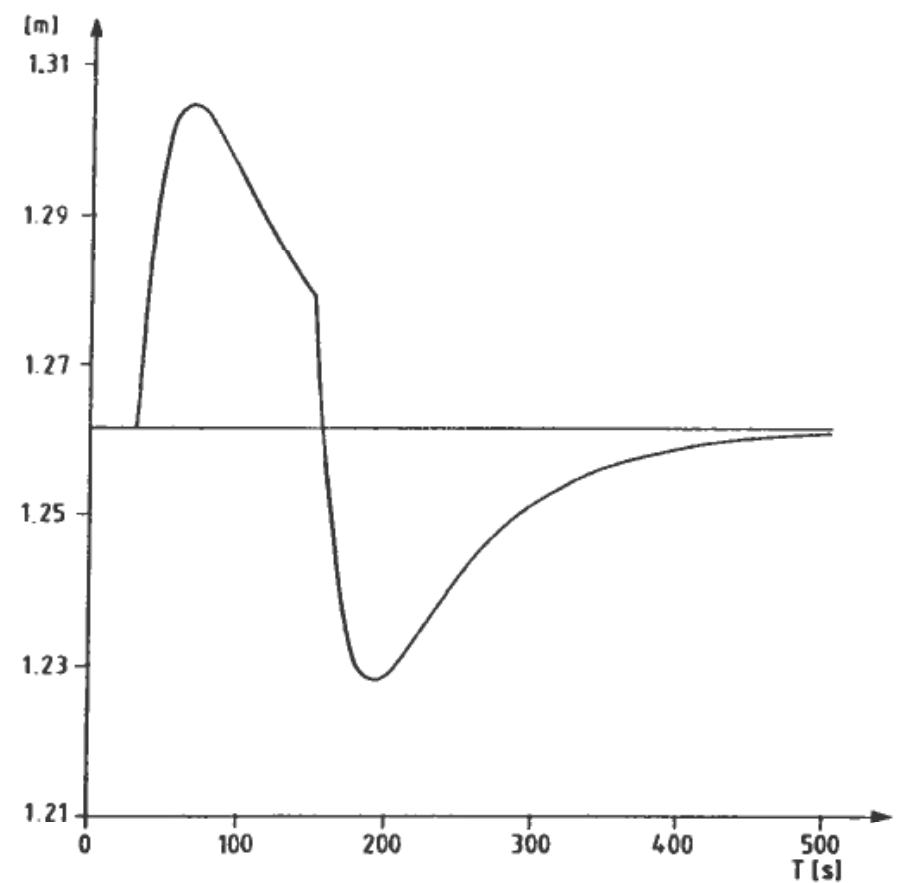

Figure 7. First separator stage liquid level and level controller reference value.

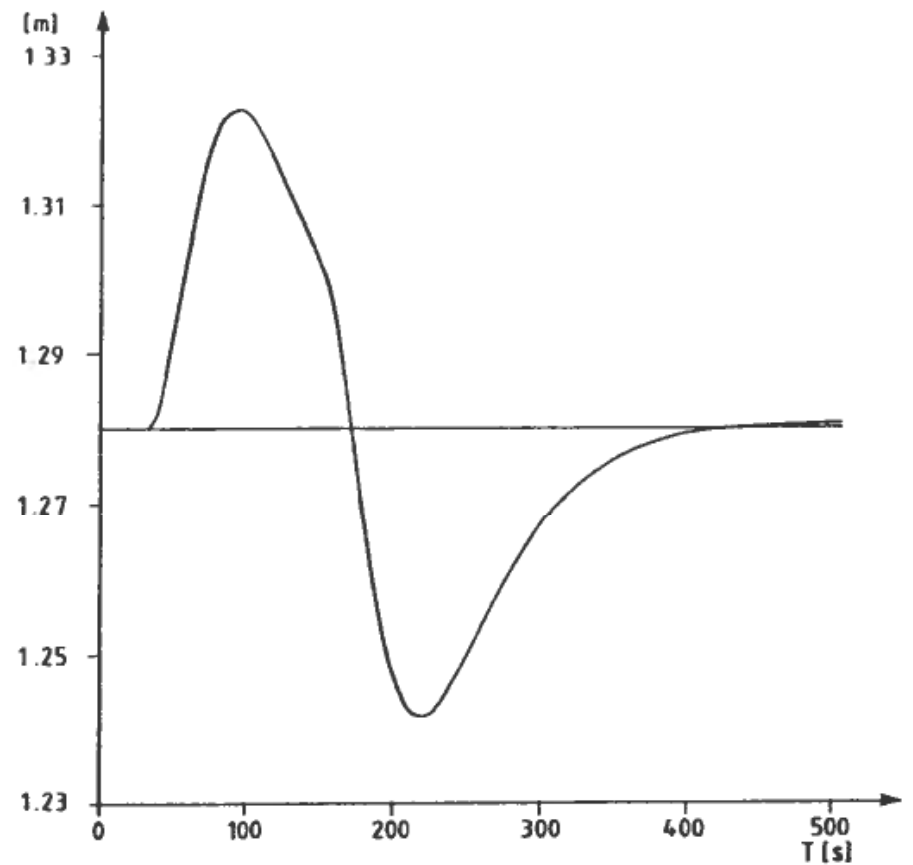

Figure 8. Second separator stage liquid level and level controller reference value. 


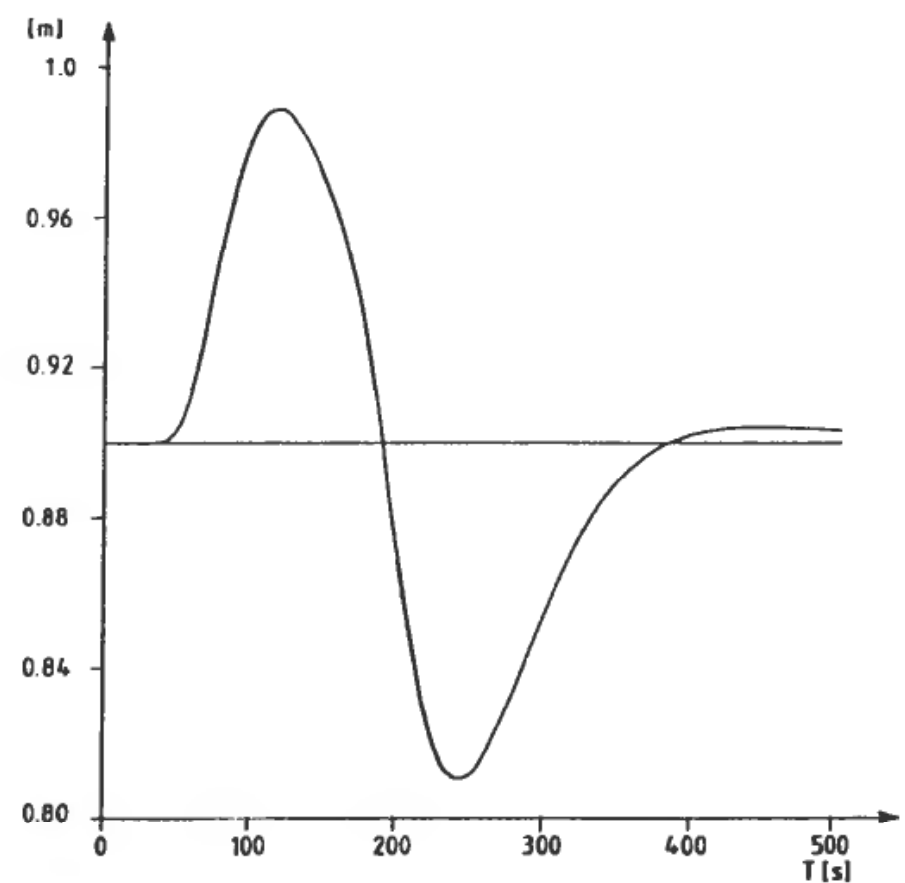

Figure 9. Third separator stage liquid level and level controller reference value.

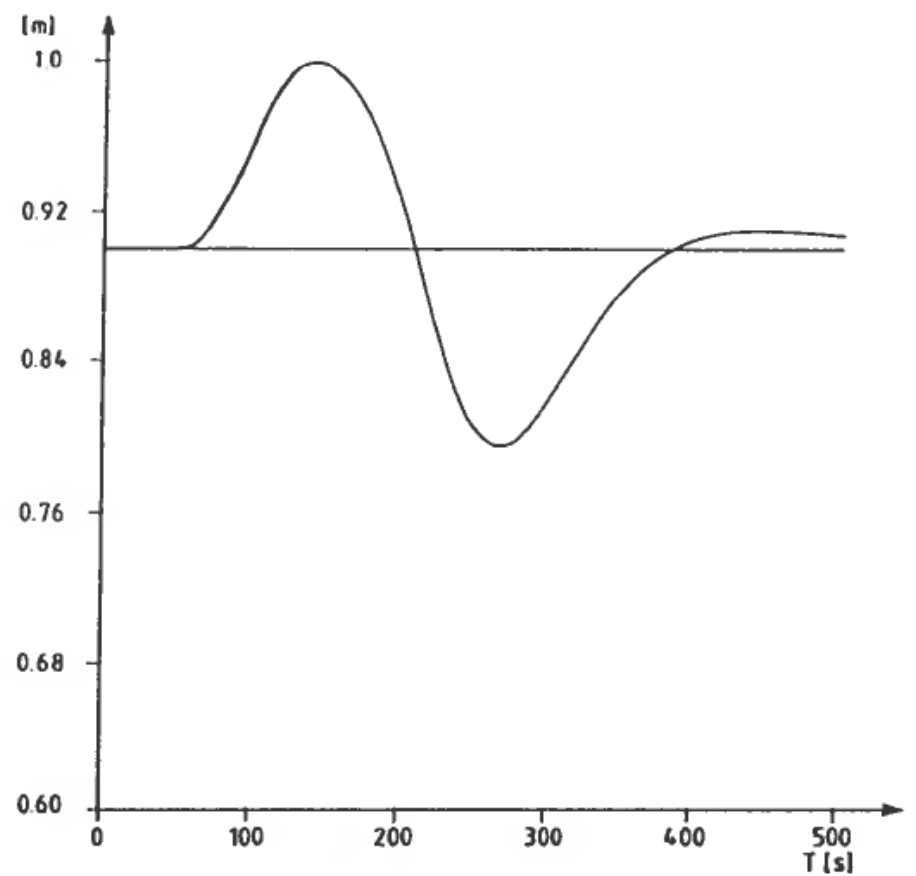

Figure 10. Fourth separator stage liquid level and level controller reference value. 


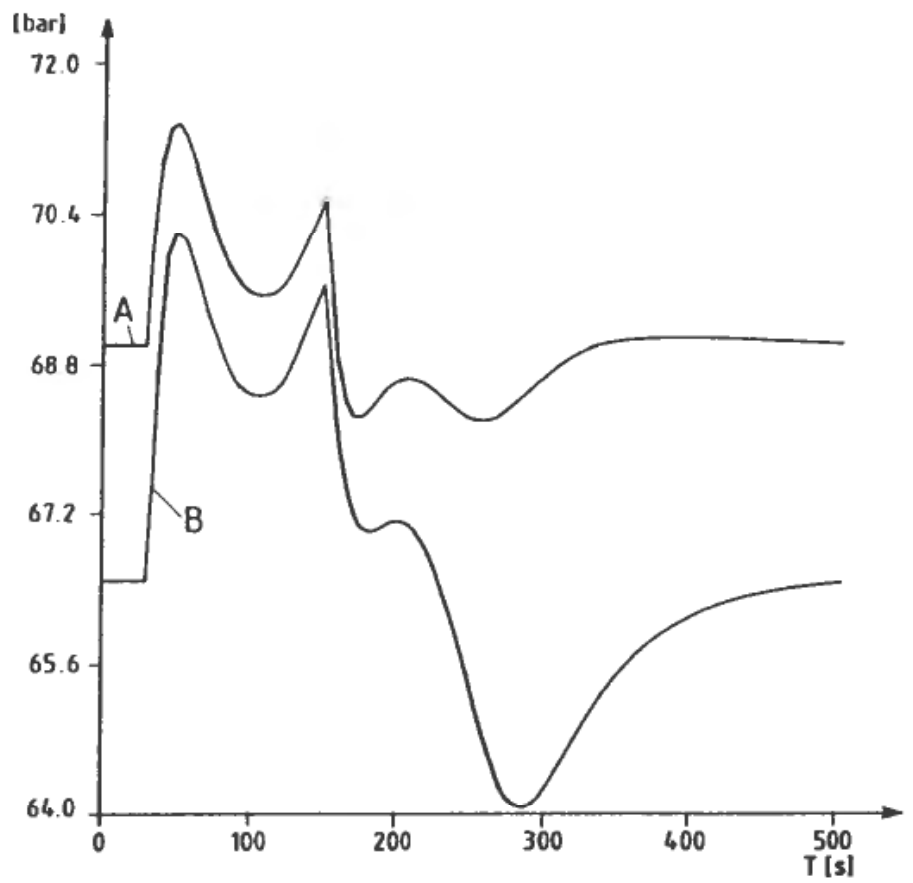

Figure 11. First separator stage pressure (A) and fourth scrubber pressure (B).

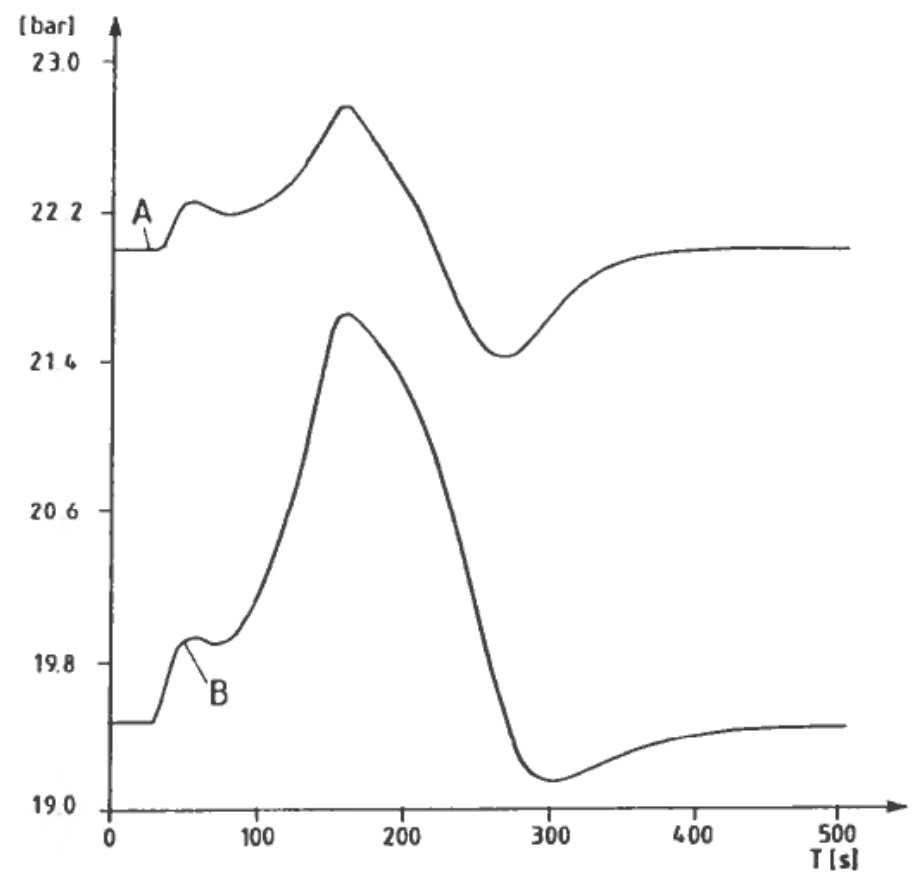

Figure 12. Second separator stage pressure (A) and third scrubber pressure (B). 


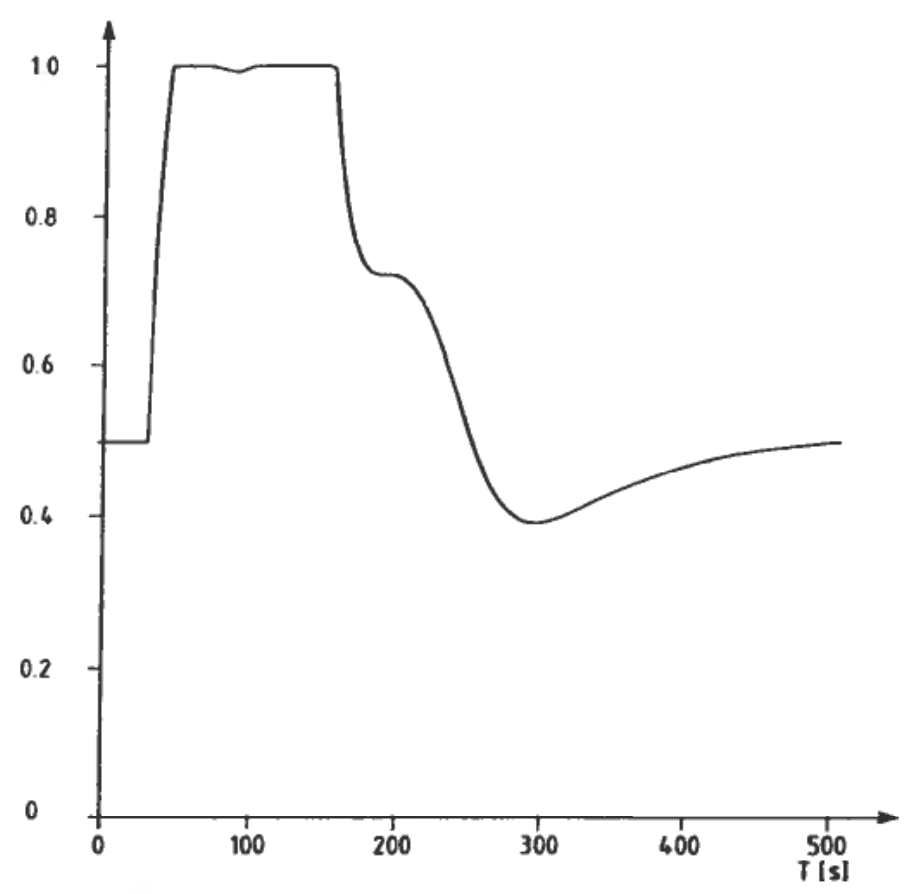

Figure 13. Control signal to first separator gas outlet control valve.

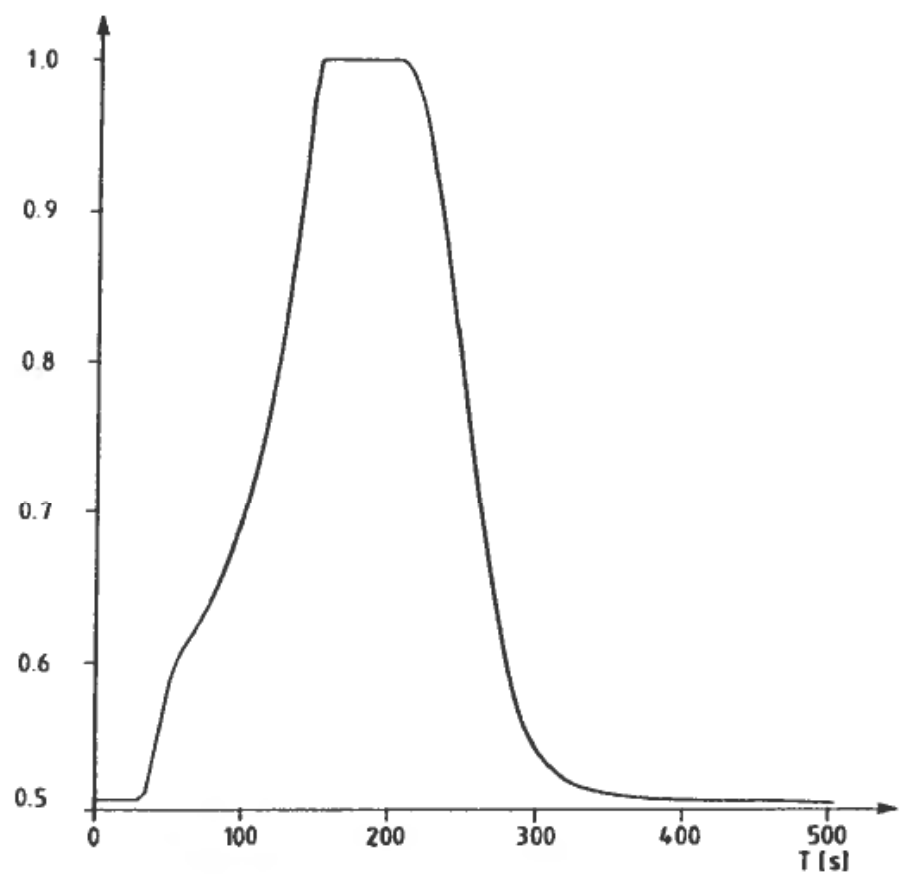

Figure 14. Control signal to second separator gas outlet control valve. 


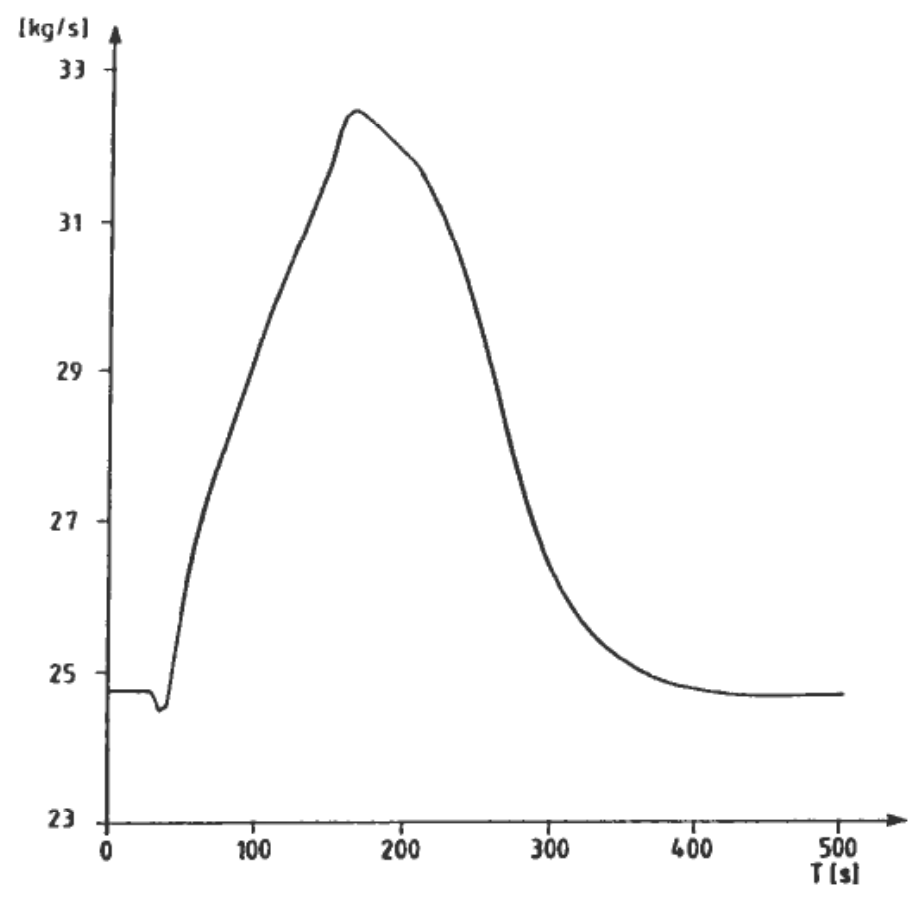

Figure 15. Mass flow rate through third compressor stage.

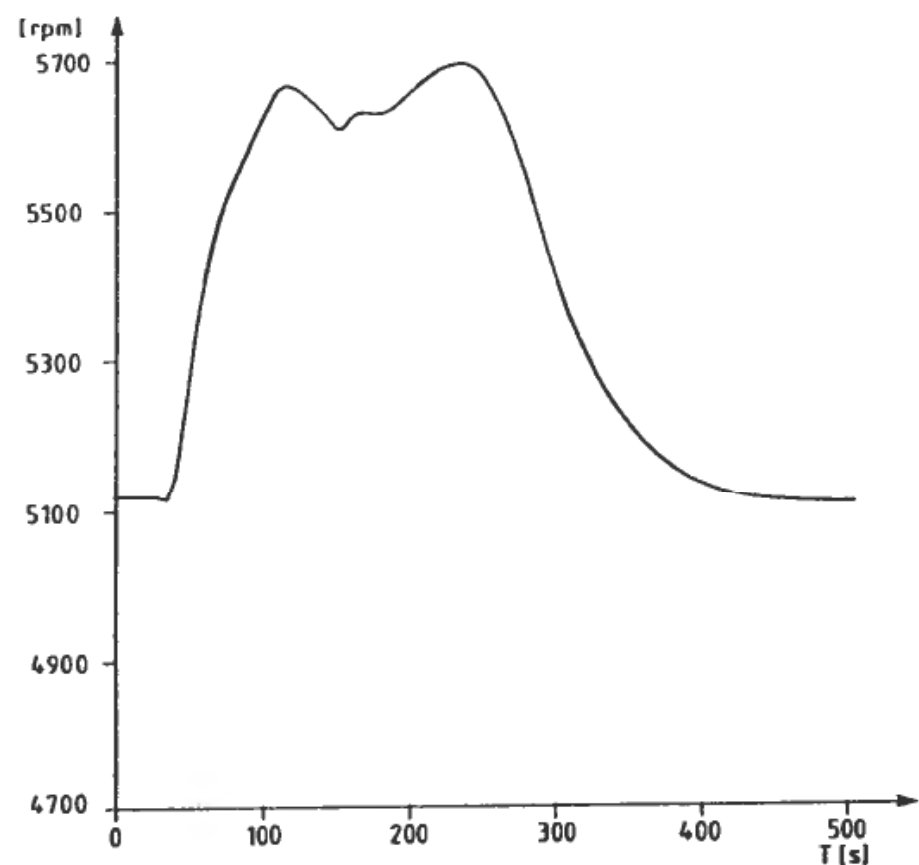

Figure 16. Gas turbine speed. 


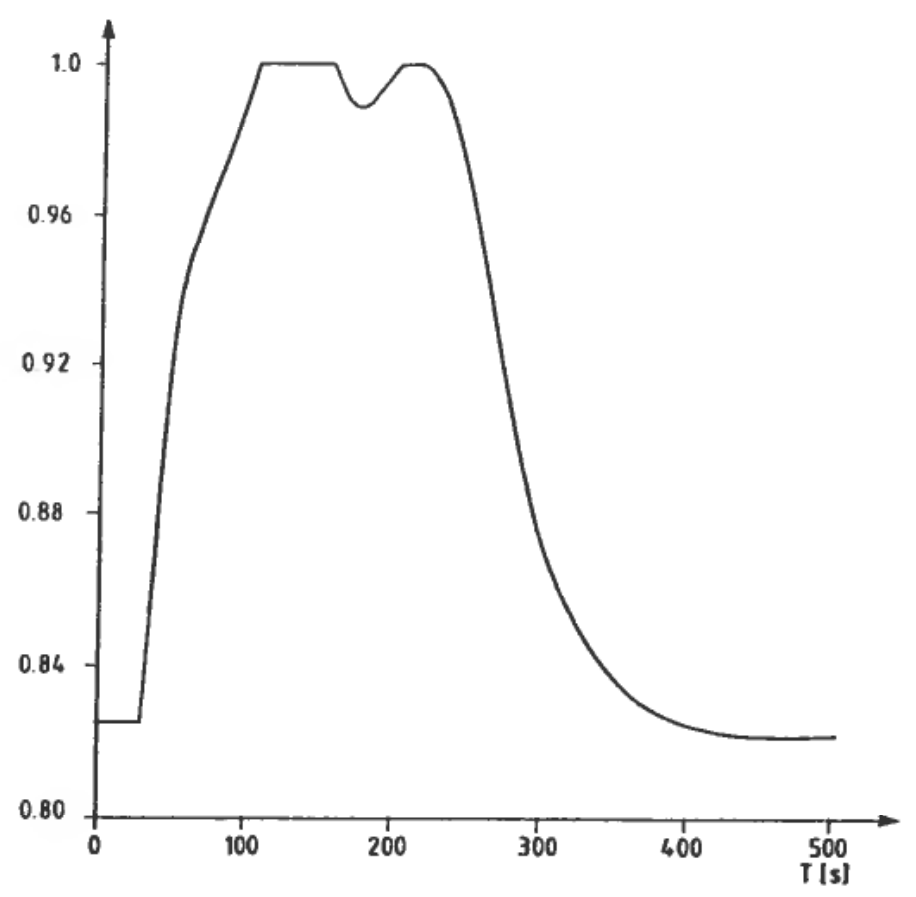

Figure 17. Gas turbine controller signal.

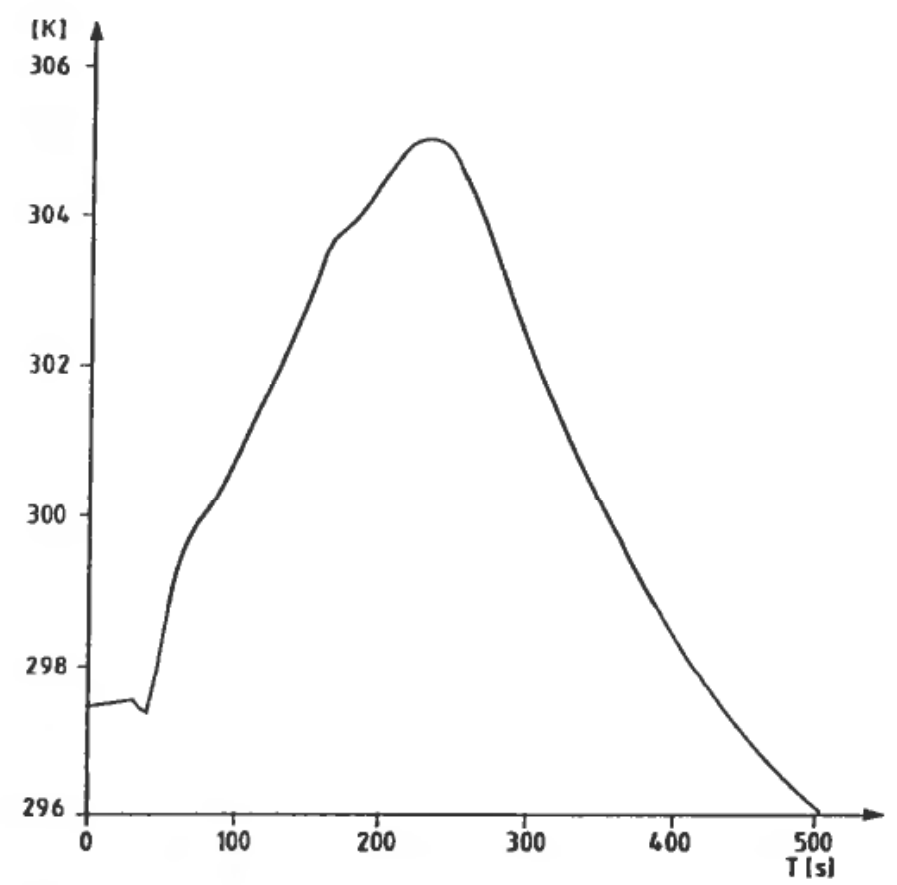

Figure 18. Third heat exchanger primary outlet temperature. 
downstream disturbances significantly. A reduction in bandwidth for the liquid level control loops will not reduce the disturbance in the outlet liquid flow rate, there will just be a delay.

The liquid level is disturbed in all separators (Figs. 7-10), but it is most serious in the third and the fourth separator stages because they are smaller than the other two. Integral time is set to 100 seconds for all liquid level controllers.

Pressure is upset in all four separators and we can easily notice that there is strong interaction between pressure in the first separator and the fourth scrubber (Fig. 11) and between pressure in the second separator and third scrubber (Fig. 12). The first and second separator stage gas outlet valves (Figs. 13-14) are saturated because the pressure across these valves is reduced during the transience.

The three compressors working on the same shaft are driven by a gas turbine. The turbine power generation is controlled by the differential pressure across the compressor module. It is important that this control loop is properly tuned in order to achieve stable operation of the module. As pressure in the third scrubber rises, the turbine generates more power and is saturated for a while (Fig. 17). There is an increase in both the gas turbine speed (Fig. 16) and the mass flow rate through the compressor module (Fig. 15).

Figure 18 shows the third stage heat exchanger outlet temperature. As heat exchangers are slow to control, temperature control does not work very well when the primary flow rate varies too quickly.

\section{Conclusion}

Dynamic process simulation is in reality the only way to study the influence of the various process disturbance and control strategies on plant performance. Usually a plant is designed with the help of a static simulation program. Even if each process unit is stable, when operated in isolation, this is not always the case when a number of process units are connected and integrated in a plant. Unstable operation can occur because of strong dynamic interaction between the process units. Dynamic simulation is an efficient tool to study different plant configurations and control strategies with respect to overall dynamic performance.

\section{Nomenclature}

$\begin{array}{cl}\text { Liquid-vapour } & \text { mass transfer } \\ X & \text { mass fraction of dissolved gas in liquid at equilibrium } \\ X_{\mathrm{q}}, X_{\mathrm{p}}, X_{\mathrm{T}} & \text { constants used in linearized calculation of } X \\ L_{\mathrm{q}}, L_{\mathrm{p}}, L_{\mathrm{T}} & \text { constants used in linearized calculation of } L \\ F & \text { inlet molar flow rate in flash calculation } \\ L & \text { liquid outlet molar flow rate } \\ V & \text { vapour outlet molar flow rate } \\ Z_{i} & \text { inlet mixture mole fraction of component no. } i \\ x_{i} & \text { outlet liquid mole fraction of component no. } i \\ y_{i} & \text { outlet gas mole fraction of component no. } i \\ p & \text { pressure }\end{array}$



$T \quad$ temperature
$k_{i} \quad$ vapour-liquid equilibrium constant for component no. $i$
$\mathrm{M}_{i} \quad$ mol. weight of component no. $i$
$M_{l} \quad$ mol. weight of liquid mixture
$\rho_{l} \quad$ liquid density
$\gamma_{s} \quad$ mass of gas in liquid solution per unit volume of liquid
$\xi \quad$ vapour mole fraction

Two-phase vapour-liquid separation

$\begin{array}{ll}R_{s, f} & \text { rate of mass transfer from liquid to vapour } \\ m_{l} & \text { mass of liquid in separator } \\ m_{v} & \text { mass of vapour in separator } \\ m_{s} & \text { mass of dissolved gas in separator } \\ w_{l, i}, w_{l, o} & \text { inlet, outlet mass flow rate of liquid } \\ w_{v, i}, w_{v, o} & \text { inlet, outlet mass flow rate of vapour } \\ w_{s, i}, w_{s, o} & \text { inlet, outlet mass flow rate of dissolved gas in liquid } \\ U & \text { internal energy } \\ H & \text { enthalpy } \\ V & \text { separator volume } \\ h_{v} & \text { specific enthalpy in vapour } \\ h_{l} & \text { specific enthalpy in liquid } \\ h_{v, i} & \text { specific enthalpy in inlet vapour } \\ h_{l, i} & \text { specific enthalpy in inlet liquid } \\ T_{q} & \text { stationary temperature } \\ P_{q} & \text { stationary pressure } \\ M_{l} & \text { liquid mol. weight } \\ M_{v} & \text { vapour mol. weight } \\ h_{e v} & \text { evaporation specific enthalpy } \\ c_{p l} & \text { liquid specific heat capacity at constant pressure } \\ c_{p v} & \text { vapour specific heat capacity at constant pressure } \\ V_{l} & \text { liquid volume } \\ z & \text { compressibility factor } \\ R & \text { universal gas constant }\end{array}$

\section{Oil-water separation}

$R_{d, w} \quad$ rate of water separation from oil phase

$l_{o} \quad$ liquid-vapour interface level

$l_{w} \quad$ water-oil interface level

$m_{d} \quad$ mass of water drops in oil phase

$m_{w} \quad$ mass of free water

$m_{o} \quad$ mass of oil

$w_{w, i}$ inlet mass flow rate of water

$w_{w, o}$ mass flow rate of water through water outlet

$w_{w, l o}$ mass flow rate of water through oil outlet

$w_{l, l o}$ total mass flow rate through oil outlet

$x_{w} \quad$ water content of liquid flow through oil outlet. 


\section{Heat exchanger}

$w_{l, i} \quad$ inlet mass flow rate of liquid

$w_{l, o} \quad$ outlet mass flow rate of liquid

$w \quad$ total mass flow rate

$w_{c} \quad$ mass flow rate of coolant

$L \quad$ liquid content in outlet flow

$T_{i} \quad$ inlet temperature

$T_{o} \quad$ outlet temperature

$T_{c, i} \quad$ coolant inlet temperature

$T_{c, o} \quad$ coolant outlet temperature

$m_{c} \quad$ mass of coolant inside heat exchanger

$m$ mass of primary medium inside heat exchanger

$c_{p} \quad$ primary medium specific heat capacity at constant pressure

$c_{c p} \quad$ coolant specific heat capacity at constant pressure

$h_{e v} \quad$ specific evaporation enthalpy

$Q \quad$ heat transfer

$U$ overall heat transfer coefficient

$A \quad$ internal surface area for heat transfer

\section{Compressor}

$f$ inlet, outlet pressure ratio

$T_{1}$ inlet temperature

$T_{2}$ outlet temperature

$w$ mass flow rate

$\eta \quad$ compressor efficiency

$k$ isentropic exponent, $k=c_{p} / c_{v}$

$n$ polytropic exponent

$h$ increase in gas specific enthalpy from inlet to outlet (polytropic head)

$z$ average compressibility factor

$B_{c}$ compressor power consumption

Gas turbine

$B_{T}$ power generated by turbine

$B_{c i}$ power consumed by compressor number $i$

$B_{F}$ friction loss

I momentum of inertia

$N$ number of rotations per minute (rpm)

$N_{0}$ normal speed (in rpm)

$T_{0}$ maximum torque at speed $N_{0}$

$T$ time lag

$u \quad$ control signal, $u \in[0,1]$

$c_{f}$ friction coefficient

Level controller
$s \quad$ variable in Laplace transform
$k_{p}$ controller proportional constant
$T_{i}$ controller integral time
$T_{n} \quad$ valve operation time lag 
$k_{v} \quad$ valve constant

$A$ liquid surface area

$\Delta p$ valve differential pressure

$\rho_{l} \quad$ liquid density

$\omega_{c} \quad$ control loop bandwidth

\section{Pressure controller}

$k_{p}$ controller proportional constant

$T_{i}$ controller integral time

$T_{v} \quad$ valve operation time lag

$k_{v} \quad$ valve constant

$V_{v}$ vapour volume

$m_{c}$ mass of liquid

$\Delta_{p} \quad$ valve differential pressure

$\rho_{v} \quad$ vapour density

\section{REFERENCES}

ARnold, K. and Stewart, M. (1984 a). Designing oil and gas production systems. How to size and select two-phase separators, World oil, November.

ARNOLD, K. and STEWART, M. (1984 b) Designing oil and gas production systems. How to size and select three-phase separators, World oil, December.

Bird, R. B., STEWART, W. E. and LightFoot, E. N. (1960). Transport Phenomena, (John Wiley \& Sons).

Callaghan, I., C., Gould, C. M., Reid, A. J. and Seaton, D. H. (1985). Crude-Oil Foaming Problems at the Sullom Voe Terminal, Journal of Petroleum Technology, December, 21112218.

CampelL, J. M. (1984). Gas Conditioning and Processing, volumes 1 and 2, Campell Petroleum Series.

LaPINA, R. P. (1982). How to use the Performance Curves to Evaluate Behaviour of Centrifugal Compressors, Chemical Engineering, January 25, 86-93.

Lydersen, A. L. (1985). Mass Transfer in Engineering Practice (John Wiley \& Sons, Chichester).

Standing, M. B. (1979). A Set of Equations for Computing Equilibrium Ratios of Crude Oil/Natural Gas System at Pressure below 1000 psia, Journal of Petroleum Technology, September, 1193-1195.

STRAROSELSKY, N., and LADIN, L. (1979). Improved Surge Control for Centrifugal Compressors, Chemical Engineering, May 21, 175-183.

Wu, F. U. (1984). Drum Separator Design, Chemical Engineering, April. 